\title{
¿EXISTE RELACIÓN ENTRE EL CONSUMO DE ALCOHOL DE LOS PADRES Y EL DE LOS ADOLESCENTES?
}

\author{
Marta García-Barba \\ barbam@uji.es \\ Cristina Giménez-García \\ Jesús Castro-Calvo \\ Juan Enrique Nebot-García \\ Rafael Ballester-Arnal
}

Salusex-Unisexsida. Dpto. Psicología Básica, Clínica y Psicobiología. Facultad de Ciencias de la Salud. Universitat Jaume I de Castellón (España).

Fecha de Recepción: 20 marzo 2018

Fecha de Admisión: 10 Abril 2018

\section{RESUMEN}

En general, se entiende que las actitudes y conductas de los padres influyen en la configuración de los hábitos de los hijos. Sin embargo, no siempre se conoce hasta qué punto podría existir dicha influencia en etapas clave como la adolescencia en las que, en principio, los pares son la fuente de referencia y ejercen un potente modelado. Por este motivo, nuestro estudio busca conocer si ciertas conductas y actitudes de padres están relacionados con el consumo y/o las actitudes que tienen sus hijos respecto al alcohol y otras drogas. Un total de 331 adolescentes (47,2\% chicos; $52,7 \%$ chicas) con edades comprendidas entre los 13 y los 18 años ( $M=14,8$; $D T=1,02)$ cumplimentaron el CIACS-2 (Ballester y Gil, 2007). La mitad de chicas y el 70\% de los chicos se han emborrachado, como mínimo, algunas veces $\left(X^{2}=12,72 ; p=, 005\right)$. Además, aproximadamente un $25 \%$ de chicos y chicas están en desacuerdo con la afirmación "no consumiría ninguna droga por miedo a sus efectos". En este marco, las actitudes paternas positivas hacia el consumo de alcohol y el propio consumo de los padres se asocian con el hecho de que los adolescentes se hayan emborrachado alguna vez, consuman alcohol los fines de semana y tengan actitudes positivas hacia el mismo. De igual forma, las actitudes positivas hacia las drogas entre los familiares se relacionan con una minimización de sus efectos adversos por parte de los adolescentes. Las conductas y actitudes de riesgo de los responsables familiares parecen modular, negativamente, las creencias y comportamientos de los propios adolescentes. Así pues, y teniendo en cuenta el papel de los padres como modelos de conducta, parece imprescindible diseñar más estrategias preventivas dirigidas a los propios responsables familiares, además de a los adolescentes.

Palabras clave: alcohol; drogas; adolescentes; padres; modelado 


\section{¿EXISTE RELACIÓN ENTRE EL CONSUMO DE ALCOHOL DE LOS PADRES Y EL DE LOS ADOLESCENTES?}

\section{ABSTRACT}

Is there a relationship between the alcohol consumption of parents and teenagers?

In general, attitudes and behaviours of parents may influence on the configuration of children's habits. However, it is unclear what extent this influence could exist on important stages such as adolescence, in which their peers are the main reference and exercise a powerful modelling. For this reason, our study explores if certain behaviours and attitudes of parents are related to the consumption and/or attitudes of their children regarding alcohol and other drugs. A total of 331 adolescents ( $47.2 \%$ boys, $52.7 \%$ girls) with ages between 13 and 18 years old $(M=14.8, S D=1.02)$ completed the CIACS-2 (Ballester y Gil, 2007). Half of the girls and $70 \%$ of the boys have been drunk, at least, once $\left(X^{2}=12.72, p=.005\right)$. In addition, approximately $25 \%$ of boys and girls disagree with the affirmation "I would not use any drug for perceiving fear of its effects". In this context, positive parental attitudes towards alcohol consumption and their parents' consumption are associated with the fact that adolescents have ever gotten drunk, consume alcohol on weekends and have positive attitudes towards it. Similarly, positive attitudes toward drugs among family members are related to adolescents' underestimate of its adverse effects. The risk behaviours and attitudes of relatives seem to modulate, negatively, adolescents' beliefs and behaviours. Therefore, and taking into account the role of parents as models, it seems essential to design more preventive strategies aimed at family responsible, as well as adolescents.

Keywords: alcohol; drugs; teenagers; parents; modeling

\section{INTRODUCCIÓN}

El consumo de alcohol y otras drogas es un problema de salud pública que incumbe a toda la sociedad. Pese a la gran cantidad de campañas preventivas realizadas, sigue siendo un problema prevalente que comienza en edades muy tempranas. En un estudio con población estadounidense, se estima en un 21\% la prevalencia de uso de alcohol en adolescentes de 13-14 años, llegando casi al 60\% en jóvenes de 17-18 años. En cuanto al consumo de marihuana/hachís estas prevalencias son del $12 \%$ y del $35 \%$ respectivamente (Johnston et al., 2016). A nivel nacional, según los últimos datos recogidos por el Plan Nacional sobre Drogas (2018), las drogas consumidas con mayor frecuencia entre adolescentes de 14 hasta 18 años son el alcohol $(75,6 \%)$, tabaco $(34,7 \%)$, cannabis $(26,3 \%)$ e hipnosedantes $(11,6 \%)$. El consumo de todas ellas se inicia a edades muy tempranas siendo, aproximadamente, los 14 años.

El alcohol es sin duda, la droga más consumida. Actualmente y en edades tempranas el patrón de consumo suele ser el binge drinking o episodios de consumo intensivo, en el que se ingieren grandes cantidades de alcohol en un periodo de tiempo reducido (habitualmente durante los fines de semana) alternado con periodos breves de abstinencia (Pérez-Milena et al., 2017). Las consecuencias negativas a corto plazo, derivadas de este tipo de consumo, abarcan problemas como intoxicaciones agudas e incluso fallecimiento debido a las mismas, accidentes de tráfico, absentismo escolar, problemas legales y consumo de otras sustancias (Kuntsche, Kuntsche, Thrul \& Gmel, 2017; Swendsen et al., 2012). Un consumo prolongado tanto de alcohol como de otras drogas, iniciado además a edades tempranas, puede tener consecuencias negativas en el desarrollo neuropsicológico que pueden afectar al aprendizaje, la memoria, fluidez verbal y funciones ejecutivas, entre otras (Fernández-Serrano, Pérez-García, Schmidt-Río-Valle \& Verdejo-García, 2010; Green et al., 2010; Sneider, Cohen-Gilbert, Crowley, Paul \& Silveri, 2013).

Ante este panorama, distintos autores han examinado aquellos factores que podrían facilitar su uso. Muchas investigaciones se centran en las motivaciones y/o características que poseen tanto las sustancias en sí, como las personas que inician y/o continúan el consumo. Respecto a los factores psicosociales estudiados en relación al consumo de sustancias tóxicas, se destacan la búsqueda de 
sensaciones, la impulsividad (Leeman, Hoff, Krishnan-Sarin, Patock-Peckham \& Potenza, 2014), y diversas motivaciones como serían la búsqueda de estados de relajación, lograr mayor resistencia física, situaciones sociales y aliviar síntomas depresivos (Boys, Marsden \& Strang, 2001). Además, se ha encontrado una importante asociación entre las motivaciones sociales/relacionales, la vinculación e integración social y el uso de diversas drogas (De la Villa Moral \& Ovejero, 2011; Kloep, Hendry, Ingebrigtsen, Glendinning \& Espnes, 2001). Moral, Rodríguez y Ovejero (2010) encontraron que los adolescentes que consumen alcohol/otras drogas presentaban un perfil diferencial respecto a los no consumidores en factores relativos a la mayor permisividad actitudinal, efecto de modelado del grupo de iguales y también paterno. Otras variables importantes en relación con la familia son la comunicación, el afecto entre padres e hijos y la autoestima (Elzo, 2014; Ochoa, Relinque, Arroyo \& González, 2015). Como vemos, la mayoría de estudios centrados en el efecto del modelado de los padres en el consumo de sustancias se centran en cómo afectan los estilos educativos, la permisividad y/o la estructura familiar sin tener en cuenta, en muchas ocasiones, el análisis de las conductas de consumo de los propios padres. Considerando la importancia del contexto familiar en el consumo de sustancias, nuestro objetivo es explorar la relación entre las actitudes y los comportamientos hacia el consumo de drogas por parte de los padres y las actitudes y conductas de consumo que realizan sus hijos.

\section{MÉTODO}

\section{Participantes}

En el estudio participaron 331 adolescentes de Enseñanza Secundaria Obligatoria, procedentes de institutos de la provincia de Castellón.

Del total, el $47,25 \%$ son chicos y el $52,75 \%$ chicas. La edad oscila entre 13 y 18 años de edad $(M=14,79 ; D T=1,02)$. La distribución por edades en función del género es homogénea $\left(\chi^{2}=1,96\right.$; $p=0,855)$. Respecto a la nacionalidad, la mayoría de ellos proceden de España $(89,2 \%)$, el $7,5 \%$ de Rumanía y el 3,3\% restantes proceden de otros países.

\section{Instrumentos utilizados}

Los participantes fueron evaluados a través de los siguientes cuestionarios:

Cuestionario de Información, Actitudes y Comportamientos relacionados con la Salud (CIACS) de Ballester y Gil (2007). Se trata de una batería formada por diferentes escalas que exploran el grado de información, las actitudes y comportamientos relacionados con diversos aspectos de la salud y estilos de vida en diferentes etapas evolutivas. El instrumento ha mostrado a nivel psicométrico una fiabilidad alta (consistencia interna $=0,890$ ) y una estabilidad temporal adecuada (testretest de 0,776) (Ballester \& Gil, 2007).

En este estudio se empleó la versión CIACS-2 compuesta por 139 ítems, de los cuales se seleccionaron 21. De estos 21, los ítems 7,8, 9, 24 y 45 (manteniendo la numeración del cuestionario original) guardan relación con el consumo de alcohol y drogas de los encuestados y de sus padres. Deben responder en función de la frecuencia con la que realizan cada acción en una escala tipo Likert de 5 puntos (de nunca a siempre/habitualmente). También se escogieron los ítems 41 al 48 y del 55 al 63 en los que se responde en función del grado de acuerdo/desacuerdo con las afirmaciones (relacionadas con las actitudes hacia el alcohol y las drogas) en una escala tipo Likert de 5 puntos (de nada hasta totalmente de acuerdo) (ver cuadro 1). 
Cuadro 1. Ítems incluidos en la evaluación de actitudes hacia el alcohol y otras drogas (manteniendo la numeración del cuestionario original)

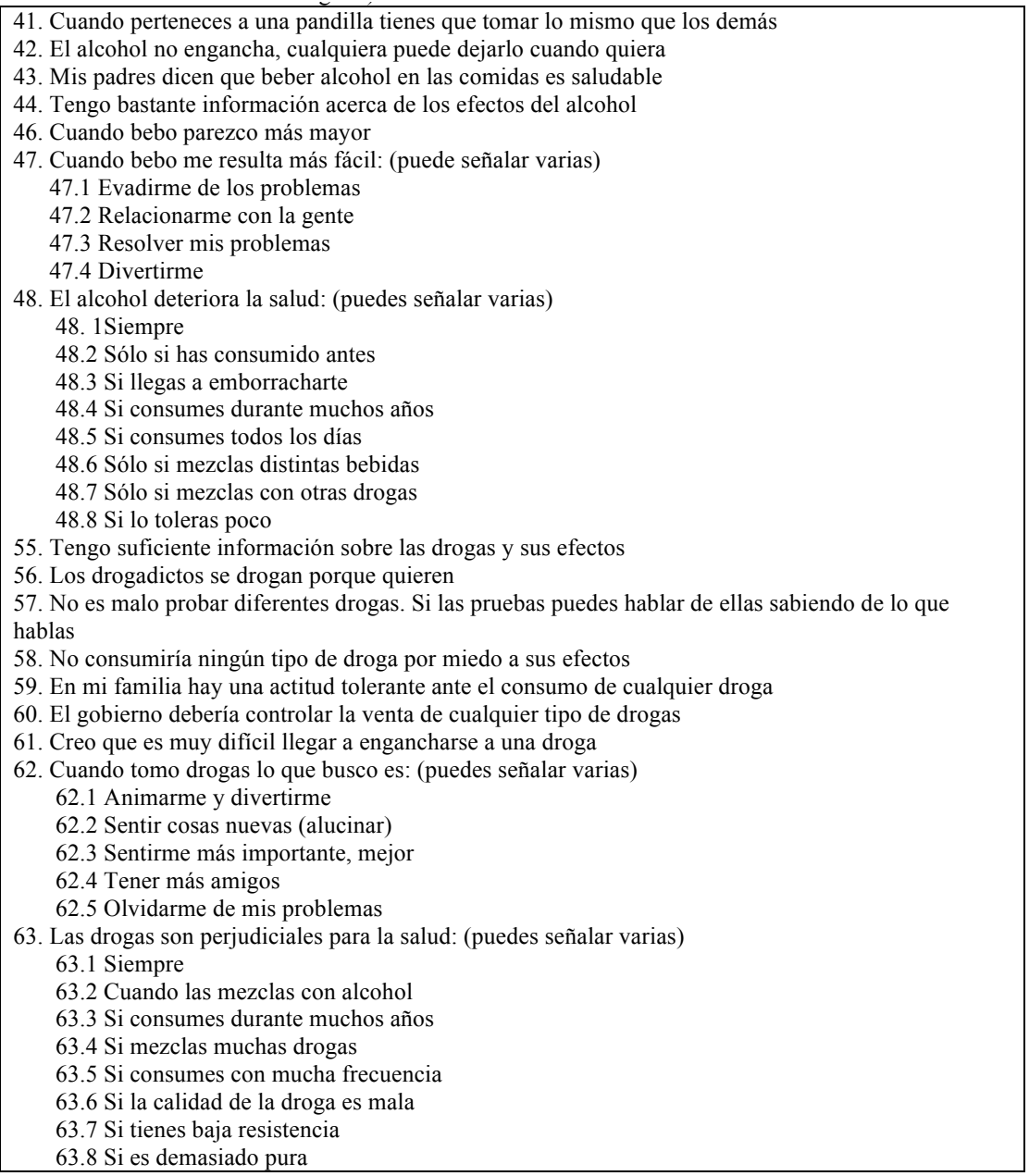

\section{PROCEDIMIENTO}

Esta investigación se encuentra dentro de un estudio más extenso que analiza estilos de vida de Ios adolescentes. Para llevarlo a cabo, se acudió a diversos centros públicos de Educación Secundaria Obligatoria pertenecientes a la Comunidad Valenciana. Inicialmente se contactó con los centros para informar sobre el objetivo de la investigación y obtener los permisos pertinentes (de los centros y de los padres de cada uno de los menores). Una vez realizado este paso y dentro del aula, se procedió a la administración del mismo recalcando el carácter anónimo, voluntario e individual del cuestionario. Posteriormente, se analizaron las respuestas a los diferentes ítems mediante el programa estadístico SPSS. Los análisis empleados fueron, además de los estadísticos des- 
criptivos, la prueba ${ }^{2}$ para comparar los resultados en función del género, y el coeficiente de correlación de Pearson, con el propósito de evaluar la relación lineal entre diferentes variables.

\section{RESULTADOS}

\section{Conductas de consumo de alcohol y drogas en los adolescentes}

Como vemos en la tabla 1, la mitad de los encuestados afirman que sus padres no les permiten beber alcohol; mientras que alrededor de un $25 \%$ nunca se lo han prohibido. Encontramos diferencias significativas entre chicos y chicas respecto al hecho de haberse emborrachado. Un tercio de ellos y casi un $20 \%$ de ellas se emborrachan con bastante frecuencia, porcentajes que aumentan considerablemente al añadir a aquellos que se han emborrachado al menos algunas veces. Más hombres que mujeres reportan tomar alcohol los fines de semana, aunque estas diferencias no son significativas. Respecto al consumo de drogas no se encuentran diferencias significativas entre géneros: entre un 4 y $9 \%$ de los chicos y chicas han consumido drogas en al menos alguna ocasión por no saber decir que no cuando sus amigos le insisten.

Tabla 1. Análisis diferenciales en conductas relacionadas con el consumo de alcohol y drogas entre hombres y mujeres.

\begin{tabular}{|c|c|c|c|c|c|c|c|c|c|}
\hline \multirow{3}{*}{ Ítem } & \multicolumn{2}{|c|}{ Nunca } & \multicolumn{2}{|c|}{ Algunas veces } & \multicolumn{2}{|c|}{ Bastantes veces } & \multicolumn{2}{|c|}{ Siempre/ } & \multirow[b]{3}{*}{$\chi^{2}(p)$} \\
\hline & \multirow[b]{2}{*}{ Hombre } & \multirow[b]{2}{*}{ Mujer } & \multirow[b]{2}{*}{ Hombre } & \multirow[b]{2}{*}{ Mujer } & \multirow[b]{2}{*}{ Hombre } & \multicolumn{3}{|c|}{ Habitualmente } & \\
\hline & & & & & & Mujer & Hombre & Mujer & \\
\hline $\begin{array}{l}\text { Mis padres no } \\
\text { me permiten } \\
\text { beber alcohol }\end{array}$ & 25,64 & 23,35 & 23,08 & 27,27 & 7,69 & 11,04 & 43,59 & 38,31 & $\begin{array}{c}1,83 \\
(, 608)\end{array}$ \\
\hline \multirow{4}{*}{$\begin{array}{l}\text { Me he } \\
\text { emborrachado } \\
\text { He consumido } \\
\text { drogas porque no } \\
\text { sé decir que no } \\
\text { cuando me } \\
\text { insisten mis } \\
\text { amigos }\end{array}$} & 32,31 & 49,38 & 36,92 & 31,88 & 20,00 & 15,63 & 10,77 & 3,13 & $\begin{array}{l}12,72 \\
(, 005)\end{array}$ \\
\hline & 91,34 & 96,25 & 5,51 & 2,50 & 2,36 & 1,25 & 0,79 & 0,00 & $\begin{array}{c}3,62 \\
(, 306)\end{array}$ \\
\hline & \multicolumn{2}{|c|}{ Nada de acuerdo } & \multicolumn{2}{|c|}{ Algo de acuerdo } & \multicolumn{2}{|c|}{$\begin{array}{l}\text { Bastante de } \\
\text { acuerdo }\end{array}$} & \multicolumn{2}{|c|}{$\begin{array}{l}\text { Totalmente de } \\
\text { acuerdo }\end{array}$} & \\
\hline & Hombre & Mujer & Hombre & Mujer & Hombre & Mujer & Hombre & Mujer & $\chi^{2}(p)$ \\
\hline $\begin{array}{l}\text { Mis amigos y yo } \\
\text { tomamos alcohol } \\
\text { los fines de } \\
\text { semana }\end{array}$ & 29,77 & 41,25 & 38,93 & 33,13 & 14,50 & 15,63 & 16,79 & 10,00 & $\begin{array}{c}5,92 \\
(, 116)\end{array}$ \\
\hline
\end{tabular}

\section{Actitudes hacia el alcohol y otras drogas en padres e hijos}

Como se muestra en la figura 1, casi un $20 \%$ está de acuerdo con que, cuando perteneces a una pandilla, tienes que tomar lo mismo que los demás (ítem 41). A más de la mitad de los participantes les resulta más fácil divertirse y relacionarse con la gente cuando beben, aunque a casi un $25 \%$ también les ayuda a evitar sus problemas (ítems 47.4;47.2;47.3). 
Figura 1. Porcentajes de respuestas de "algo" a "totalmente de acuerdo" a los ítems sobre actitudes hacia el alcohol.

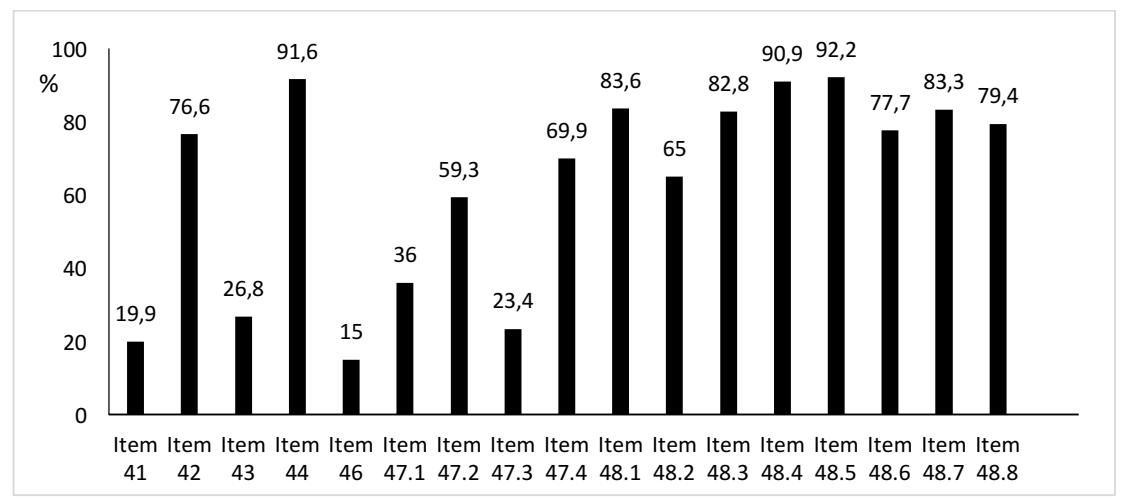

Finalmente, gran parte de los participantes opina que el alcohol deteriora siempre la salud, sobre todo cuando se hace con alta frecuencia, prolongado en el tiempo y mezclado con otras drogas (ítems 48.1;48.3;48.4;48.5 y 48.7). Sin embargo, algo más de un cuarto de participantes dice que sus padres consideran saludable el consumo de alcohol en las comidas (ítem 43). En la figura 2 encontramos porcentajes similares, pero referentes a las actitudes hacia las drogas. La mayoría de los participantes consideran que tienen información suficiente sobre éstas y sus efectos (ítem 55), más de un $70 \%$ no consumiría ninguna droga por miedo a sus efectos (ítem 58) y un cuarto de los adolescentes creen que es bueno probar las drogas para hablar de ellas sabiendo del tema (ítem 57). Sin embargo, casi un $80 \%$ opina que los drogadictos se drogan porque quieren (ítem 56) y aproximadamente la mitad considera que es muy difícil llegar a engancharse a una droga (ítem 61). Las motivaciones más frecuentes para el consumo de drogas en estos sujetos son animarse y divertirse, olvidarse de sus problemas y sentir cosas nuevas (alucinar) (ítems 62.1;62.5 y 62.2). En cuanto a las actitudes de los padres, un $30 \%$ considera que en su familia hay una actitud tolerante hacia el consumo de cualquier droga (ítem 59).

Figura 2. Porcentajes de respuestas de "algo" a "totalmente de acuerdo" a los ítems sobre actitudes hacia otras drogas.

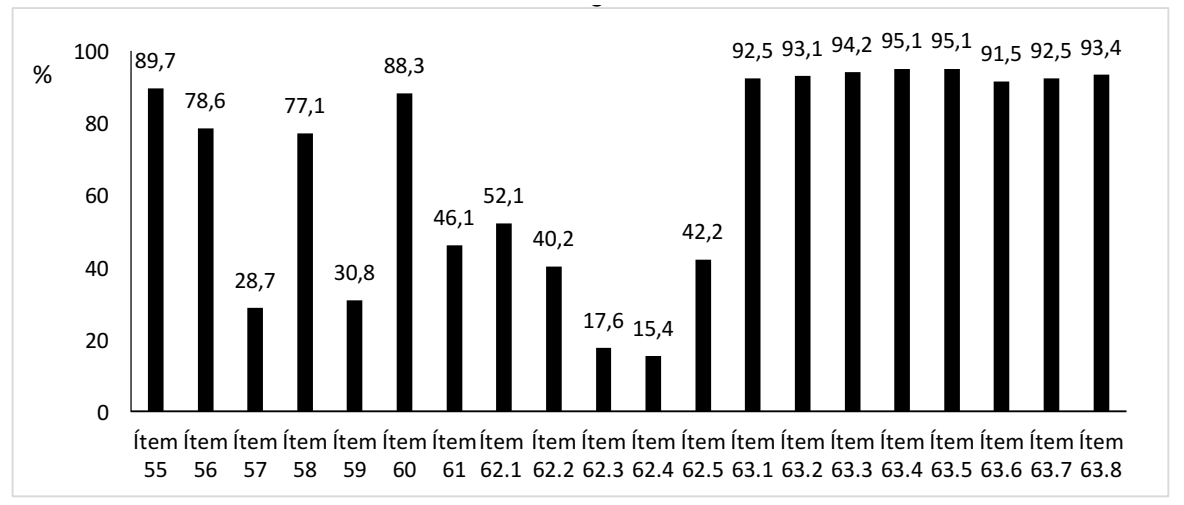


Asociación entre las conductas y actitudes de los padres y las conductas y actitudes de sus hijos hacia el consumo de alcohol y otras drogas.

Tabla 2. Correlaciones entre las conductas y las actitudes de los padres hacia el consumo de alcohol y las conductas y actitudes de sus hijos.

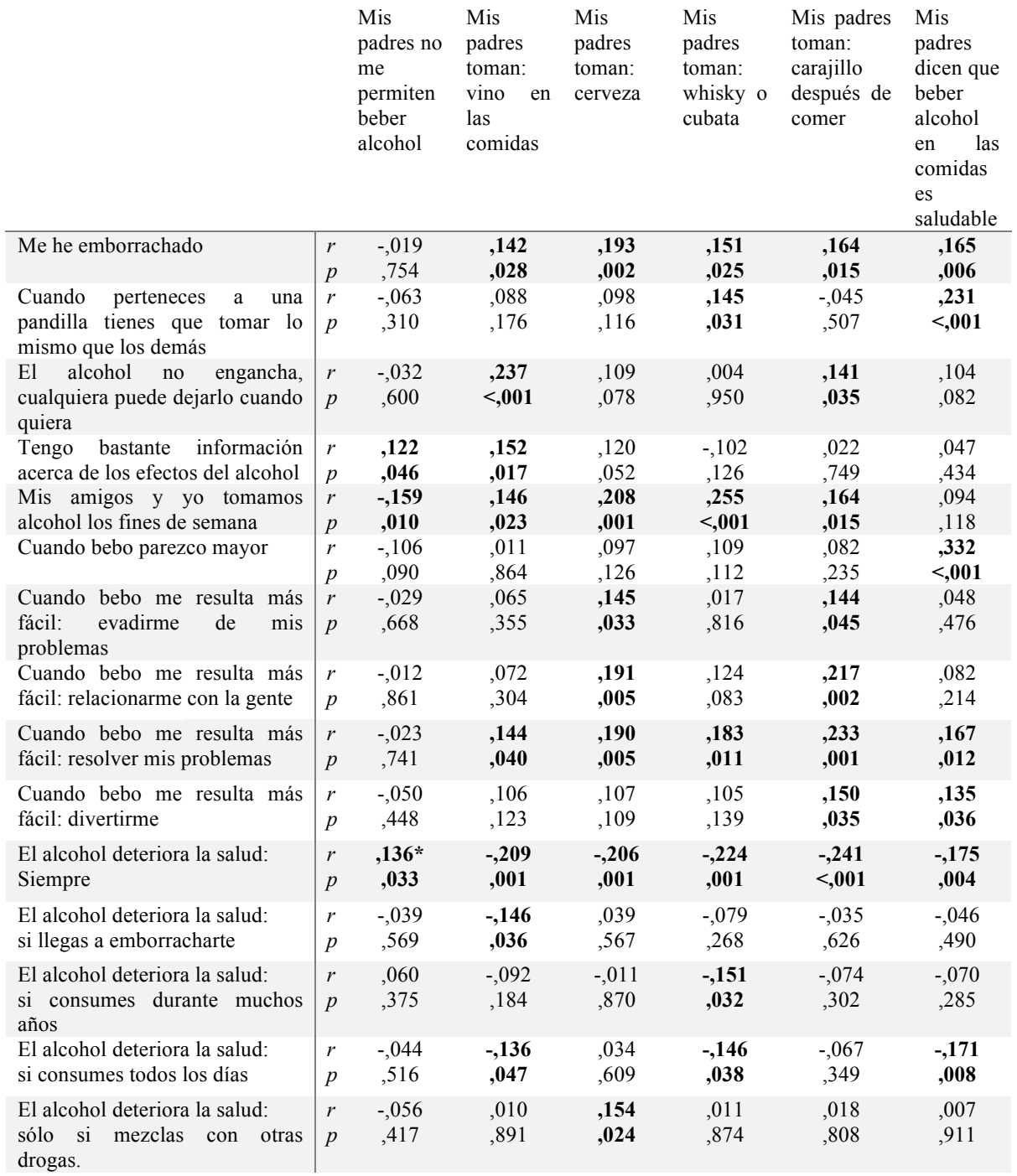

Como indican los resultados (ver tabla 2), el consumo de alcohol de los padres y las actitudes positivas frente al alcohol se relacionan con diversas conductas de consumo y con actitudes tolerantes hacia el mismo por parte de los adolescentes. El consumo por parte de los padres de vino, cerveza, whisky/cubata y carajillo se relaciona de forma positiva con haberse emborrachado y beber 
los fines de semana con amigos por parte de los adolescentes. Mientras que se revela una asociación contraria si les prohíben consumir alcohol. Además, cuanto más consumo de este tipo de bebidas por parte de los padres, menos creencia tienen sus hijos de que el alcohol deteriore siempre la salud y más reforzante les resulta el alcohol como medio de resolver sus problemas 0 evadirse de ellos y de relacionarse con gente. El hecho de que los padres verbalicen que beber alcohol en las comidas es saludable también se relaciona positivamente con haberse emborrachado y con ciertas creencias como la de tener que consumir lo mismo que tu grupo de iguales, parecer más mayor cuando se consume alcohol y distintos aspectos reforzantes del consumo de alcohol. Estas verbalizaciones se relacionan de forma negativa en la valoración sobre la peligrosidad del consumo de alcohol.

Finalmente, las actitudes tolerantes hacia las drogas por parte de los padres se han visto relacionadas únicamente con la minimización de los efectos adictivos de este tipo de sustancias (Tabla 3).

Tabla 3. Correlaciones entre las actitudes hacia las drogas de los padres y las actitudes de sus hijos hacia las mismas.

\begin{tabular}{l|cc} 
& \multicolumn{2}{c}{$\begin{array}{l}\text { En mi familia hay una actitud tolerante ante el consumo } \\
\text { de cualquier droga }\end{array}$} \\
\hline & $r$ & $P$ \\
Los drogadictos se drogan porque quieren & $\mathbf{0 , 1 4 8}$ & $\mathbf{0 , 0 1 5}$ \\
Creo que es muy difícil llegar a \\
engancharse a una droga
\end{tabular}

\section{CONCLUSIONES Y DISCUSIÓN:}

Los padres ejercen un papel muy importante en el desarrollo de sus hijos como modelos de todo tipo de conductas. Si bien es cierto que en la adolescencia, etapa donde suele iniciarse el consumo de sustancias, el grupo de iguales ejerce bastante influencia, los padres siguen desempeñando un papel muy importante en su desarrollo (Azpiazu, Esnaola \& Sarasa, 2015). El objetivo de este estudio era comprobar si las conductas de consumo de los padres y sus actitudes se relacionan con las actitudes y conductas de sus hijos.

Nuestros resultados indican que, coincidiendo con los datos del último informe del Plan Nacional Sobre Drogas (2018), el consumo de alcohol en estas edades se da con bastante frecuencia. Como esperábamos y en concordancia con los datos de otros estudios (Alfonso, Huedo-Medina \& Espada, 2009; Ballester \& Gil, 2009; Janssen, Mathijssen, van Bon-Martens, Van Oers \& Garretsen, 2014) el consumo de alcohol de los padres, así como las actitudes permisivas hacia éste guardan relación con el consumo de alcohol en los adolescentes y con actitudes positivas hacia esta sustancia. Respecto a los resultados referentes a otro tipo de drogas, únicamente hemos encontrado relación entre las actitudes de tolerancia hacia el consumo por parte de los padres y la baja percepción del riesgo adictivo de las mismas. Esto puede deberse a varios factores como la baja aceptación social que tienen el resto de drogas comparadas con el alcohol, sustancia cuyo consumo está muy extendido y valorado positivamente en nuestra sociedad. Esto va ligado también a la baja frecuencia del consumo de drogas comparadas con el alcohol, además de la no asociación de sustancias como el tabaco con el concepto de droga.

Estudios similares también recalcan la importancia de la influencia de los familiares (concretamente padres y hermanos) no solo en el consumo de alcohol, sino también en el de otras sustancias (Ruiz-Risueño, Ruiz-Juan \& Zamarripa, 2012). De hecho, los hallazgos de Moral, Rodríguez y Ovejero (2010) apuntan que el papel del modelado de los padres pronostica el consumo tanto de alcohol como otro tipo de drogas. 
Estos resultados cabría tenerlos en cuenta, a la luz de ciertas limitaciones que presenta este estudio. La más importante es que reducimos el ámbito de estudio a unos cuántos ítems que, aunque nos den información general, difícilmente pueden describir el amplio abanico que envuelve al consumo de sustancias.

En definitiva, encontramos cierta relación entre las actitudes y conductas hacia las drogas por parte de los padres y las de sus hijos, que podría ayudar a confirmar la relación existente entre los estilos de vida de los responsables familiares y los adolescentes. En este sentido, sería importante profundizar en la relación de estas variables. Teniendo en cuenta todo lo anterior, parece imprescindible diseñar más estrategias preventivas dirigidas a los propios responsables familiares, además de a los adolescentes.

\section{REFERENCIAS}

Alfonso, J. P., Huedo-Medina, T. B., \& Espada, J. P. (2009). Factores de riesgo predictores del patrón de consumo de drogas durante la adolescencia. Anales de psicología, 25(2), 330-338.

Azpiazu, L., Esnaola, I., \& Sarasa, M. (2015). Capacidad predictiva del apoyo social en la inteligencia emocional de adolescentes. European journal of education and psychology, 8(1), 23-29.

Ballester, R. \& Gil, M.D. (2007). Cuestionario de Información, Actitudes y Comportamientos relacionados con la Salud (CIACS-I, II y III): Estudio psicométrico. V World Congress of Behavioral \& Cognitive Therapies, Barcelona 12, 13 y 14 de Julio de 2007.

Ballester, R. \& Gil, M.D (2009). ¿Por qué los jóvenes se dan atracones de alcohol los fines de semana? Estudio sobre creencias y actitudes relacionadas con ese patrón de consumo y diferencias de género. Revista de Psicopatología y Psicología Clínica, 1, 25-35.

Boys, A., Marsden, J., \& Strang, J. (2001). Understanding reasons for drug use amongst young people: a functional perspective. Health education research, 16(4), 457-469.

De la Villa Moral, M., \& Ovejero, A. (2011). Consumo abusivo de alcohol en adolescentes españoles: tendencias emergentes y percepciones de riesgo. Universitas Psychologica, 10(1), 71-87.

Fernández-Serrano, M. J., Pérez-García, M., Schmidt-Río-Valle, J., \& Verdejo-Garcia, A. (2010). Neuropsychological consequences of alcohol and drug abuse on different components of executive functions. Journal of Psychopharmacology, 24(9), 1317-1332.

Green, A., Garrick, T., Sheedy, D., Blake, H., Shores, E. A., \& Harper, C. (2010). The effect of moderate to heavy alcohol consumption on neuropsychological performance as measured by the repeatable battery for the assessment of neuropsychological status. Alcoholism: Clinical and Experimental Research, 34(3), 443-450.

Janssen, M. M., Mathijssen, J. J., van Bon-Martens, M. J., Van Oers, H. A., \& Garretsen, H. F. (2014). A qualitative exploration of attitudes towards alcohol, and the role of parents and peers of two alcohol-attitude-based segments of the adolescent population. Substance abuse treatment, prevention, and policy, 9(1), 20-30.

Johnston, L. D., O'Malley, P. M., Miech, R. A., Bachman, J. G., \& Schulenberg, J. E. (2016). Monitoring the Future national survey results on drug use, 1975-2015: Overview, key findings on adolescent drug use. Ann Arbor: Institute for Social Research, The University of Michigan.

Kloep, M., Hendry, L. B. L., Ingebrigtsen, J. E., Glendinning, A. \& Espnes, G. A. (2001). Young people in 'drinking' societies? Norwegian, Scottish and Swedish adolescents'perception of alcohol use. Health Education Research, 16(3), 279-291.

Kuntsche, E., Kuntsche, S., Thrul, J., \& Gmel, G. (2017). Binge drinking: health impact, prevalence, correlates and interventions. Psychology \& health, 32(8), 976-1017. 
Leeman, R. F., Hoff, R. A., Krishnan-Sarin, S., Patock-Peckham, J. A., \& Potenza, M. N. (2014). Impulsivity, sensation-seeking, and part-time job status in relation to substance use and gambling in adolescents. Journal of Adolescent Health, 54(4), 460-466.

Moral, M.V., Rodríguez, F.J. \& Ovejero, A. (2010). Correlatos psicosociales del consumo de sustancias psicoactivas en adolescentes españoles. Salud Pública de México, 52, 406-415.

Ochoa, G. M., Relinque, C. S., Arroyo, G. D. M., \& González, M. E. V. (2015). El consumo de alcohol en adolescentes: El rol de la comunicación, el funcionamiento familiar, la autoestima y el consumo en la familia y amigos. Búsqueda, 2(14), 45-61.

Pérez-Milena, A., de Dios Redondo-OImedilla, M., Martínez-Fernández, M. L., Jiménez-Pulido, I., Mesa-Gallardo, I., \& Leal-Helmling, F. J. (2017). Cambios en el consumo alcohólico de riesgo en población adolescente en la última década (2004-2013): una aproximación cuanti-cualitativa. Atención Primaria, 49(9), 525-533.

Plan Nacional sobre Drogas. (2018). Encuesta estatal sobre uso de drogas en estudiantes de enseñanzas secundarias (ESTUDES) 2016-2017, España. Madrid: Delegación del Gobierno para el Plan Nacional sobre Drogas. Recuperado de:http://www.pnsd.msssi.gob.es/profesionales/ sistemasInformacion/sistemalnformacion/pdf/ESTUDES_2016_Presentacion.pdf

Ruiz-Risueño, J., Ruiz-Juan, F., \& Zamarripa, J. I. (2012). Alcohol y tabaco en adolescentes españoles y mexicanos y su relación con la actividad físico-deportiva y la familia. Revista Panamericana de Salud Pública, 31(3), 211-220.

Sneider, J. T., Cohen-Gilbert, J. E., Crowley, D. J., Paul, M. D., \& Silveri, M. M. (2013). Differential effects of binge drinking on learning and memory in emerging adults. Journal of addiction research \& therapy, 7. Doi: 10.4172/2155-6105.S7-006

Swendsen, J., Burstein, M., Case, B., Conway, K. P., Dierker, L., He, J., \& Merikangas, K. R. (2012). Use and abuse of alcohol and illicit drugs in US adolescents: Results of the National Comorbidity Survey-Adolescent Supplement. Archives of general psychiatry, 69(4), 390-398. 